\title{
CASO 9-2014: Peritonitis por Rahnella aquatilis en un paciente en Diálisis Peritoneal Crónica Ambulatoria
}
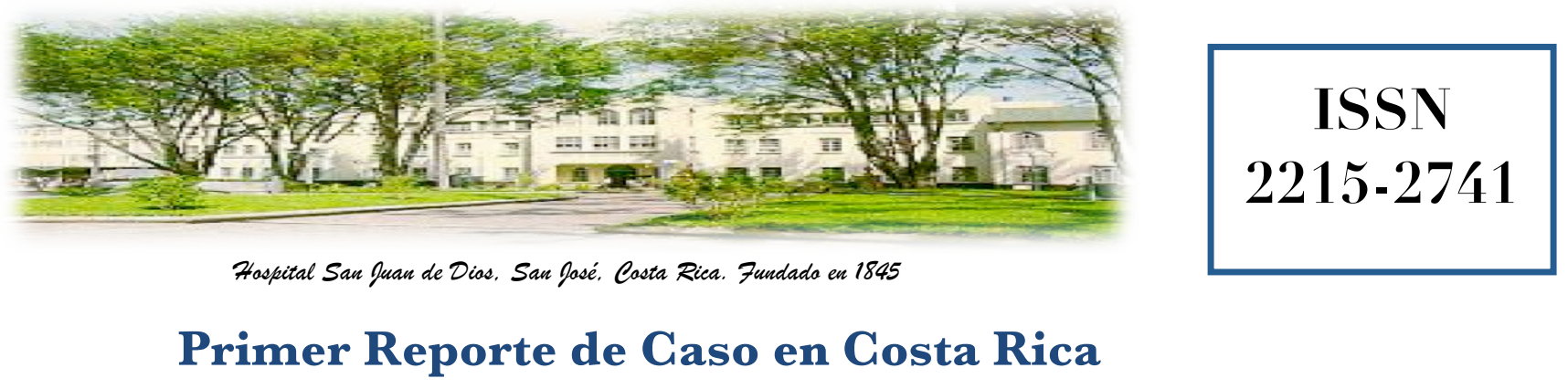

$\begin{array}{ll}\text { Recibido: } & 26 / 03 / 2014 \\ \text { Aceptado: } & 28 / 08 / 2014\end{array}$

\author{
Saúl G. Quirós Cárdenas ${ }^{1}$ \\ Elvira Segura Retana ${ }^{2}$ \\ Manuel Antonio Villalobos Zúñiga ${ }^{3}$
}

\footnotetext{
${ }^{1}$ Médico Residente del Postgrado de Medicina Interna de la Universidad de Costa Rica. Médico Residente de la Sección de Medicina del Hospital San Juan de Dios.

${ }^{2}$ Microbióloga Especialista en Bacteriología. Laboratorio de Bacteriología del Hospital San Juan de Dios.

${ }^{3}$ Médico Especialista en Infectología y Medicina Interna. Hospital San Juan de Dios. Correo electrónico: manuel701@gmail.com
}

\section{RESUMEN}

Masculino de 34 años, quien se encuentra en el programa de Diálisis Peritoneal Crónica Ambulatoria (DPCA) del Hospital San Juan de Dios (HSJD), debido a una Insuficiencia Renal Crónica (IRC) Estadio 5; quien presentó una peritonitis bacteriana asociada a DPCA. En el líquido peritoneal se aisló Rahnella aquatilis. Se observó adecuada resolución del caso tras cobertura antibiótica con Cefotaxime intraperitoneal. Este es el primer caso reportado en Costa Rica de peritonitis bacteriana causada por Rahnella aquatilis en un paciente de DPCA.

\section{PALABRAS CLAVE}

Diálisis Peritoneal. Peritonitis. Rahnella aquatilis.

\section{ABSTRACT}

A 34-year-old male patient, on Continuous Ambulatory Peritoneal Dialysis (CAPD) due to Chronic Kidney Disease (CKD) Stage 5, who presented a bacterial peritonitis associated with CAPD, isolating Rahnella aquatilis in the peritoneal fluid. Appropriate resolution of the case was observed after antibiotic coverage with intraperitoneal Cefotaxime. This is the first bacterial peritonitis caused by Rahnella aquatilis in a patient of CAPD reported in Costa Rica.

\section{KEY WORDS}

Peritoneal Dialysis. Peritonitis. Rahnella aquatilis. 


\section{CASO CLÍNICO}

Masculino de 34 años vecino de Ciudad Colón, San José. Portador de drepanocitosis, hipertensión arterial e IRC Estadio 5. Es paciente del servicio de Nefrología del HSJD y en el mes de mayo de 2013 se le colocó un catéter flexible para DPCA tipo Tenckhoff. Tiene el antecedente de dos episodios de peritonitis: el primero de ellos en el mes de mayo de 2013 durante la realización de una diálisis peritoneal aguda y el segundo en el mes de junio del mismo año, aislándose en líquido peritoneal un Staphylococcus haemolyticus como agente causal.

Consultó el 25 de noviembre de 2013 por historia de dolor abdominal, sensación febril y el hallazgo de turbidez en el efluente de la diálisis peritoneal. A su ingreso al Servicio de Emergencias Médicas del HSJD, se documentó temperatura en $37.8{ }^{\circ} \mathrm{C}$ y dolor abdominal difuso. Se realizó el análisis de una muestra de líquido peritoneal, que evidenció la presencia de 8150 leucocitos $/ \mathrm{mm}^{3}$, de ellos el $95 \%$ fueron neutrófilos y el 5\% linfocitos; además de 100 eritrocitos $/ \mathrm{mm}^{3}$; una glucosa en $191 \mathrm{mg} / \mathrm{dl}$, proteínas en $<1.5 \mathrm{~g} / \mathrm{dl}$, deshidrogenasa láctica en $35 \mathrm{U} / 1 \mathrm{y}$ amilasa en $16 \mathrm{U} / 1$; se envió además una muestra para cultivo en una botella de hemocultivo.

El paciente presentaba leucocitosis en $20.1 \times 10^{3}$, con $88 \%$ de neutrófilos, $7 \%$ de linfocitos y $4 \%$ de bandas; una hemoglobina en $8.7 \mathrm{~g} / \mathrm{dl}$ con un hematocrito en $25 \%$. El 26 de noviembre fue ingresado a la Unidad de DPCA del Servicio de Nefrología y se inició cobertura antibiótica empírica con vancomicina 1 gramo intraperitoneal y titulación posterior.

El día 30 de noviembre se recibió el reporte preliminar del cultivo de líquido peritoneal donde se observó un bacilo Gram negativo; por lo que de decidió suspender la cobertura antibiótica con vancomicina e iniciar tratamiento con Cefotaxime intraperitoneal, la dosis empleada fue de $250 \mathrm{mg}$ en cada intercambio de diálisis peritoneal (4 intercambios por día). Dos días después se recibió el reporte definitivo del cultivo, en el cual se constata el crecimiento de Rahnella aquatilis con la respectiva prueba de sensibilidad antibiótica (Tabla 1). El catéter de Tenckoff fue preservado en este caso. La muestra se cultivó en dos ocasiones subsecuentes obteniendo el mismo aislamiento ya documentado.

Tabla 1. Resultados de la prueba de susceptibilidad a antibióticos de Rahnella aquatilis

\begin{tabular}{|c|c|c|c|}
\hline Antibiótico & Resultado & Antibiótico & Resultado \\
\hline $\begin{array}{l}\text { Amoxacilina- } \\
\text { Clavulánico }\end{array}$ & $\mathbf{S}$ & Ciprofloxacina & $\mathbf{S}$ \\
\hline Ampicilina & $\mathbf{R}$ & Cloranfenicol & $\mathbf{S}$ \\
\hline Cefalotina & $\mathbf{R}$ & Gentamicina & $\mathbf{S}$ \\
\hline Cefotaxima & $\mathbf{S}$ & Amikacina & $\mathbf{S}$ \\
\hline Cefoxitima & $\mathbf{S}$ & Meropenem & $\mathbf{S}$ \\
\hline Cefepime & $\mathbf{S}$ & Imipenem & $\mathbf{S}$ \\
\hline Ceftazidima & $\mathbf{S}$ & TMP-SMX & $\mathbf{S}$ \\
\hline $\begin{array}{l}\text { S: Sensible. } \\
\text { Sulfametoxazo }\end{array}$ & Resist & TMP-SMX: & Tripetoprim \\
\hline
\end{tabular}

El caso presentó buena evolución clínica tras la modificación de la cobertura antibiótica; las características macroscópicas del líquido mejoraron en las primeras 72 horas de tratamiento con Cefotaxime y el análisis de líquido peritoneal del 3 de diciembre mostró solamente 20 leucocitos $/ \mathrm{mm}^{3}$. El paciente se egresó tras completar 14 días de tratamiento antibiótico; con evidencia de resolución de su proceso infeccioso, tanto desde el punto de vista clínico como en lo que respecta a análisis de laboratorio

\section{DISCUSIÓN}

La diálisis peritoneal está asociada con un alto riesgo de infección peritoneal, en particular la DPCA. Los pacientes que desarrollan peritonitis usualmente presentan un líquido peritoneal de aspecto turbio y dolor abdominal. Algunos casos esporádicos pueden presentarse sin turbidez en el efluente, por lo que la peritonitis debe contemplarse dentro del diagnóstico diferencial de los pacientes en diálisis peritoneal con dolor abdominal y fiebre. Los cocos Gram positivos son la causa más frecuente de peritonitis asociada a diálisis peritoneal a nivel mundial ${ }^{(1,2)}$.

Existen algunas variantes en cuanto a los agentes causales de acuerdo a la distribución geográfica; siendo por ejemplo a nivel de la India más frecuente el observar la presencia de bacilos Gram negativos como agentes causales de este tipo de peritonitis $^{(1)}$. Pese a lo anterior, tal y como se observa en la Tabla 2, existe un amplio espectro 
de agentes causales cuando se trata de peritonitis asociada a DPCA.

En el caso específico de este paciente, el cual se presentó con historia de dolor abdominal asociado a fiebre, además del hallazgo de líquido peritoneal de aspecto turbio; se documentó la presencia de más de 8000 leucocitos $/ \mathrm{mm}^{3}$ con un franco predominio de neutrófilos $(95 \%)$ en la muestra analizada; cumpliendo con lo establecido por las Guías de la Sociedad Internacional de Diálisis Peritoneal; la cuales establecen que debe haber un conteo leucocitario $\geq 100$ leucocitos $/ \mathrm{mm}^{3}$ con al menos $50 \%$ de neutrófilos ${ }^{(1)}$.

Tabla 2. Etiología bacteriana de la peritonitis asociada a Diálisis Peritoneal Crónica Ambulatoria

\begin{tabular}{|c|c|}
\hline Grupo & Ejemplos \\
\hline $\begin{array}{c}\text { Cocos Gram } \\
\text { Positivos }\end{array}$ & $\begin{array}{l}\text { Staphylococcus epidermidis } \\
\text { Staphylococcus aureus } \\
\text { A y } \beta \text {-haemolytic Streptococus } \\
\text { Micrococci }\end{array}$ \\
\hline $\begin{array}{c}\text { Bacilos } \\
\text { Gram } \\
\text { Negativos }\end{array}$ & $\begin{array}{l}\text { Enterobacteriaceae } \\
\text { Pseudomonas aeroginosa } \\
\text { VRE } \\
\text { Escherichia coli } \\
\text { Klebsiella oxytoca } \\
\text { Acinectobacter sp } \\
\text { Serratia marcescens } \\
\text { Enterococci }\end{array}$ \\
\hline Hongos & $\begin{array}{l}\text { Candida albicanns } \\
\text { Candida parapsilosis } \\
\text { Candida glabrata } \\
\text { Neosartorya hiratsukae } \\
\text { Aspergillus fumigatus }\end{array}$ \\
\hline \multicolumn{2}{|l|}{ Anaerobios } \\
\hline $\begin{array}{l}\text { Gérmenes } \\
\text { inusuales }\end{array}$ & $\begin{array}{l}\text { Mycobacteria sp } \\
\text { Mycobacteria NO tuberculosa de rápido } \\
\text { crecimiento } \\
\text { Listeria monocytogenes } \\
\text { Serratia marcenseus } \\
\text { Bordetella bronchiseptica } \\
\text { Corynebacterium ulcerans } \\
\text { Acanthoamoeba }\end{array}$ \\
\hline
\end{tabular}

VRE: Enterococos resistentes a vancomicina.

Fuente: Akoh JA. World J Nephrol 2012

La cobertura antibiótica empírica en el caso de peritonitis asociada a DPCA, de acuerdo a lo recomendado con las guías internacionales; debe incluir cobertura adecuada para cocos Gram positivos con un antibiótico como vancomicina o una cefalosporina y además debe contemplarse un antibiótico con adecuada acción sobre microorganismos Gram negativos, pudiendo utilizarse una cefalosporina de tercera generación o un aminoglicósido $^{(1)}$. La elección siempre debe estar orientada con base en el perfil de resistencia bacteriano que se conozca a nivel local ${ }^{(2)}$. Se prefiere de forma generalizada la administración de la terapia antibiótica por vía intraperitoneal y no por vía intravenosa; utilizando dosis continuas o intermitentes de acuerdo con la farmacocinética del antibiótico utilizado ${ }^{(1)}$.

De forma rutinaria, a nivel del HSJD, se elige la cefalotina y la gentamicina intraperitoneal como la cobertura antibiótica empírica ante la sospecha o la confirmación de un caso de peritonitis asociada a DPCA, esto en ausencia de cultivos positivos. En este caso en particular se optó por utilizar vancomicina dado el antecedente de una peritonitis por Staphylococcus haemolyticus menos de seis meses antes del episodio actual. Dicha cobertura antibiótica se modificó optando por el uso de cefotaxime intraperitoneal ante el hallazgo de un bacilo Gram negativo en la muestra de líquido peritoneal. Al momento de realizar la tipificación del microorganismo gran negativo se identificó como Rahnella aquatilis.

La familia Enterobacteriaceae contempla gran cantidad de especies, ahora mejor identificadas tanto a nivel experimental como clínico debido a la disposición de tecnología aplicada, una de estas especies que ahora podemos detectar es la Rahnella aquatilis.

Dicho micoorganismo fue identificado por Gavi$\mathrm{ni}^{(3)}$ y colaboradores en el año 1976, proveniente una muestra ambiental francesa de agua dulce y se le llamó enterobacteria del "grupo H2". Posteriormente en 1979 Izard y colaboradores ${ }^{(4)}$, mediante el uso de técnicas de hibridación del ADN, la clasificaron como un nuevo miembro de la familia Enterobacteriaceae. Se le dio dicho nombre en reconocimiento a Otto Rahn, un bacteriólogo alemán que la descubrió. La primera muestra clínica con una Rahnella aquatilis provino de un paciente quemado y fue analizada en el CDC en $1985^{(5)}$. En el año 2012 se determinó la secuencia del genoma de la bacteria ${ }^{(6)}$.

Resulta difícil distinguir dicha bacteria de otros miembros de la familia Enterobacteriaceae. Se pueden utilizar sistemas de identificación automatizada como Vitek 2 Compac de Biomerieux. Rahnella aqualitis es un bacilo Gram negativo, que presenta una serie de reacciones bioquímicas 
imprescindibles para su identificación (Tabla 3) ya que la colonia a simple vista no se diferencia en nada de cualquier otra enterobacteria (Figura $1)$.

Tabla 3. Características bioquímicas de Rahnella aquatilis

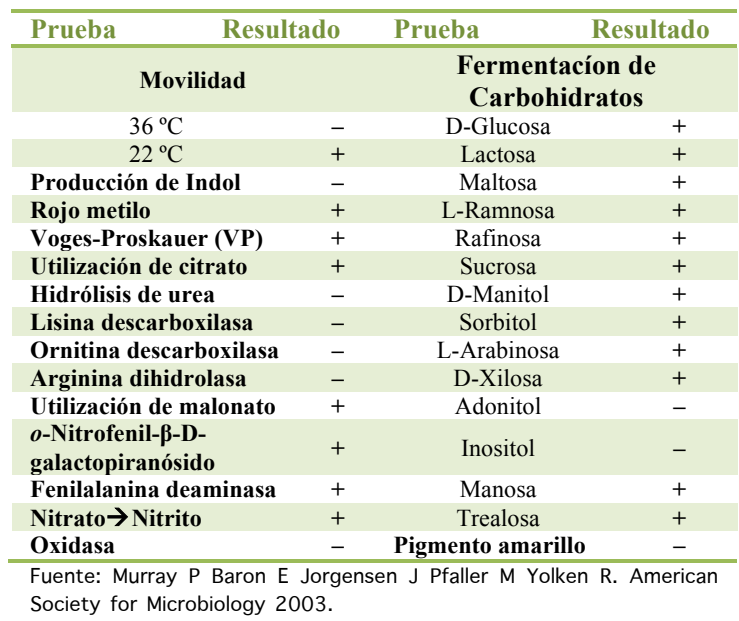

Muchos aislamientos son naturalmente resistentes a las aminopenicilinas, las carboxipenicilinas y las cefalosporinas de primera generación, por la producción de enzimas tipo ß-lactamasas ${ }^{(7,8)}$. Además de que presentan resistencia innata similar a la familia Enterobacteriaceae a los macrólidos, glicopéptidos, oxacilina, rifampicina y recientemente a fosfomicina ${ }^{(7,9,10)}$. Rahnella aqualitis tienen susceptibilidad a los aminoglucósidos, quinolonas, carbapenemas, TMP-SMX $\mathrm{y}$ algunas cefalosporinas, como en el presente caso (Tabla 1). En general se puede decir que no es una bacteria que por el momento exhiba resistencia clínicamente importante.

La cepa aislada del líquido peritoneal del caso que se presenta, tiene características en cultivo muy similares a cualquier miembro de la familia Enterobacteriaceae, crece en 18 a 24 horas a $35^{\circ} \mathrm{C}$, en atmósfera de $\mathrm{CO}_{2}$, es una bacteria lactosa positiva y se observa como bacilo Gram negativo (Figura 1).

Esta bacteria no es un patógeno humano habitual, la mayoría de las muestras analizadas en la literatura provienen de cultivos ambientales del agua; sin embargo, desde 1985 se han reportado casos de infección en el hombre. El primero de ellos como ya se relató fue en un quemado ${ }^{(5)}$ y desde entonces se ha descrito de forma aislada casos en forma de traqueítis bacteriana ${ }^{(11)}$, infección urinaria ${ }^{(12)}$, bacteremia ${ }^{(13)}$, endocarditis ${ }^{(14)}$, infección del sitio quirúrgico ${ }^{(15)}$ y como infección nosocomial por insumos contaminados ${ }^{(16,17)}$.

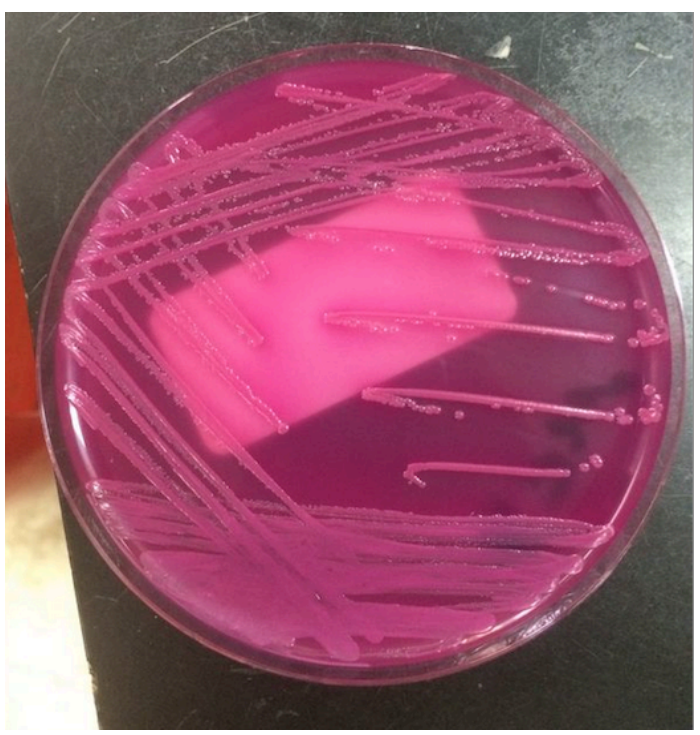

Figura 1. Cultivo del líquido peritoneal con la enterobacteria Rahnella aquatilis en agar MacKonkey.

Fuente: Laboratorio de Bacteriología del Hospital San Juan de Dios.

Aunque los reportes en la literatura no son exhaustivos, no se consigna la infección por Rahnella aquatilis como causa directa de muerte en ningún caso y otro dato que llama la atención es que la mayoría de los casos descritos son en pacientes inmunocomprometidos: pacientes con VIH, un paciente trasplantado renal y de médula ósea, un niño con cardiopatía congénita y drepanocitosis, entre otros ${ }^{(18-21)}$. No se reconoce como patógeno usual en IRC, lo cual se refuerza por la ausencia de reportes como el presente en la literatura mundial.

En Costa Rica el único reporte que conocemos es del año 2000 en el Hospital Nacional de Niños, donde se identificó Rahnella aquatilis en una paciente de 34 años con una fístula enterocutánea y de una niña de 4 años con infección urinaria, sin ninguna condición predisponente 
descrita, ambos casos con una buena respuesta clínica con los antibióticos suministrados ${ }^{(22)}$.

Estos demuestra con pocas excepciones, que la bacteria Rahnella aquatilis se comporta clínicamente como oportunista y que su virulencia parece ser baja. También esta bacteria puede comportarse como un agente de infección nosocomial y asociado a dispositivos intravasculares, como en el caso que se reporta. En general son pocos los reportes de infección por este microorganismo, lo que le agrega valor a reportes como el presente, en lo que respecta al conocimiento de nuevos agentes patógenos y su interacción clínica con los pacientes en los escenarios cada vez más complejos de la medicina moderna.

\section{CONCLUSIONES}

De esta forma se reporta el inusual caso de infección por Rahnella aquatilis en un paciente en DPCA, presentándose como una peritonitis bacteriana, según la literatura se trata del primer caso mundial reportado con esta forma de presentación. Asimismo, es el segundo reporte realizado en Costa Rica y pasaron 14 años entre el anterior y el actual. En este caso se trató de una infección de baja virulencia que respondió al tratamiento antibiótico, todo esto aumenta el conocimiento acerca de nuevas bacterias que afectan al hombre en diferentes maneras, como lo es la enterobacteria Rahnella aquatilis.

\section{BIBLIOGRAFÍA}

1. Kam-Tao P Chun C Piraino B et al. Peritoneal Dialysis Related Infections Recommendations: 2010 Update. International Society for Peritoneal Dialysis. Perit Dial Int. 2010; 30:393-423.

2. Akoh JA. Peritoneal dialysis associated infections: An update on diagnosis and management. World J Nephrol 2012;1(4): 106-122.

3. Gavini F Ferragut C Lefebvre B et al. Taxonomic study of enterobacteria belonging or related to the genus Enterobacter. Ann Microbiol(paris). 1976;127B:317-335.

4. Izard D Gavini F Trinel PA et al. Rahnella aquatilis, a new member of the Enterobacte- riaceae. Ann Microbiol (Paris). 1979:130, 163-177.

5. Farmer JJ Davis BR Hickman-Brenner FW et al. Biochemical identification of new species and biogroups of Enterobacteriacea isolated from clinical specimens. J Clin Microbiol. 1985;21(1):46-76.

6. Martínez RJ Bruce D Detter C et al. Complete Genome Sequence of Rahnella aquatilis CIP 78.65. J Bacteriol. 2012;194(11): 3020-3021.

7. Murray P Baron E Jorgensen J Pfaller M Yolken R. Manual of Clinical Microbiology. American Society for Microbiology (Washington D.C.). 2003: 639.

8. Lartigue MF Nordmann $\mathrm{P}$ Edelstein MV Cuzon G Brisse S Poirel L. Characterization of an extended-spectrum class A $\beta$-lactamse from a novel enterobacterial species taxonomically related to Rahnella spp. / Ewingella spp. J Antimicrob Chemother. 2013;68:1733-1736.

9. Stock I Gruger T Wiedemann B. Natural antibiotic susceptibility of Rahnella aquatilis an $R$. aquatilis-related strains. J Chemother. 2000;12:30-39.

10. O'Hara K Chen J Shigenobu F et al. Appearance of fosfomycin resistant Rahnella aquatilis clinical isolates in Japan. Microbios 1998;95:109-111.

11. Harrell LJ Cameron ML O'Hara CM. Rahnella aquatilis, an unusual Gram-Negative Rod Isolated from the Bronquial Washing of a Patient with Acquired Immunodeficiency Syndrome. J Clin Microbil 1989:7(27): 1671-1672.

12. Alballaa SR Qadri MH Al-Furayh O AlQatary K. Urinary tract infection due to Rahnella aquatilis in a renal transplant patient. J Clin Microb 1992;11(30):2948-2950.

13. Funke G Rosner H. Rahnella aquatilis Bacteremia in an HIV-Infected Intravenous Drug Abuser. Diagn Microbiol Infect Dis. 1995;22:293-296.

14. Matsukura H Katayama K Kitano $\mathrm{N}$ et al. Case reports: Infective Endocarditis caused by Unusual Gram-Negative Rod, Rahnella aquatilis. Pediatr Cardiol. 1996;17:108-111.

15. Maraki S Samonis G Marnelakis E Tselentis Y. Surgical wound infection caused by Rahnella aquatilis. J Clin Microb 1994;32(11): 2706-2708. 
16. Chang Ch Jeong J Hwan J Lee E Chul H. Rahnella aquatilis Sepsis in a Immunocompetent Adult. J Clin Microb. 1999;37(12): 4161-4162.

17. Caroff N Chamoux C Gallou L et al. Two epidemiologically related cases of Rahnella aquatilis bacteremia. Eur J Clin Microbiol Infect Dis 1998; 17: 349-52.

18. Gaitán J y Bronze MS. Infection caused by Rahnella aquatilis. Am J Med Sci. 2010; 339(2):577-579.

19. Tash K. Case reports: Rahnella aquatilis Bacteremia from a suspected urinary source. J Clin Microb. 2005;43(5):25262528.

20. Carinder JE Chua JD Corales RB Taege AJ Procop GW. Rahnella aquatilis bacteremia in a patient with relapsed acute lymphoblastic leukemia. Scand J Infect Dis. 2001; 33(6):471-473.

21. Hoppe JE Herter M Aleksic S Klingebiel T Niethemmer D. Catheter-related Rahnella aquatilis bacteremia in a pediatric bone marrow transplant recipient. J Clin Microbiol. 1993;31(7):1911-1912.

22. Herrera ML Vargas A Herrera JF. Primeras identificaciones de Rahnella aquatilis en Costa Rica. Rev Med Hosp Nac Niños. 2000;35(1).

\section{CONFLICTO DE INTERÉS}

Los autores declaran que no existió ningún conflicto de interés en el presente reporte. 